\title{
Enhanced accumulation of reduced glutathione by Scopoletin improves survivability of dopaminergic neurons in Parkinson's model
}

\author{
Priyadarshika Pradhan ${ }^{1}$, Olivia Majhi ${ }^{1}$, Abhijit Biswas $^{1}$, Vinod Kumar Joshi $^{2}$ and Devanjan Sinha (1)
}

\begin{abstract}
Parkinson's disease (PD) is a neuromotor disorder, primarily manifested by motor anomalies due to progressive loss of dopaminergic neurons. Although alterations in genetic factors have been linked with its etiology, exponential accumulation of environmental entities such as reactive oxygen species (ROS) initiate a cyclic chain reaction resulting in accumulation of cellular inclusions, dysfunctional mitochondria, and overwhelming of antioxidant machinery, thus accelerating disease pathogenesis. Involvement of oxidative stress in PD is further substantiated through ROS induced Parkinsonian models and elevated oxidative markers in clinical PD samples; thereby, making modulation of neuronal oxidative load as one of the major approaches in management of PD. Here we have found a potent antioxidant moiety Scopoletin (Sp), a common derivative in most of the nootropic herbs, with robust neuroprotective ability. Sp increased cellular resistance to ROS through efficient recycling of GSH to prevent oxidative damage. The Sp treated cells showed higher loads of reduced glutathione making them resistant to perturbation of antioxidant machinery or neurotoxin $\mathrm{MPP}^{+}$. Sp could restore the redox balance, mitochondrial function, and prevented oxidative damage, leading to recovery of dopaminergic neural networks and motion abilities in Drosophila genetic model of PD. Our data also suggest that Sp, in combination increases the therapeutic potency of L-DOPA by mitigating its chronic toxicity. Together, we highlight the possible ability of Sp in preventing oxidative stress mediated loss of dopaminergic neurons and at the same time enhance the efficacy of dopamine recharging regimens.
\end{abstract}

\section{Introduction}

Parkinson's disease (PD) is a progressive neurological disorder manifested clinically in four cardinal motor symptoms; muscular rigidity, bradykinesia, resting tremor, and postural imbalance occurring at later stages ${ }^{1}$. These chronic attributes are generally associated with selective degeneration of dopaminergic neurons, corollary depigmentation of substantia nigra pars compacta (SNpc) and concomitant accumulation of $\alpha$-synuclein in insoluble inclusions called Lewy bodies ${ }^{2,3}$. Most of PD cases

Correspondence: Devanjan Sinha (devanjan@bhu.ac.in)

'Department of Zoology, Institute of Science, Banaras Hindu University, Varanasi 221005, India

${ }^{2}$ Department of Dravyaguna, Institute of Medical Sciences, Banaras Hindu University, Varanasi 221005, India

Edited by P.G. Mastroberardino are sporadic and only a minor subset of overall PD cases is associated to be familial ${ }^{4,5}$. Although $\alpha$-synuclein is frequently implicated in PD pathogenesis, other proteins such as PTEN induced putative kinase 1 (PINK1), Parkin, DJ-1, leucine-rich repeat kinase 2 (LRRK2) have been strongly linked to familial forms of $\mathrm{PD}^{6,7}$.

Given the progressive nature of the disorder, disease pathogenesis is mainly triggered by continual processes such as geometric accumulation of oxidative radicals brought about by cellular inclusions and mitochondrial dysfunction $^{8-10}$. For example, PINK1 and Parkin loss of function impair mitophagy and cause accumulation of damaged mitochondria, leading to enhanced electron leakage and formation of superoxides ${ }^{11-13}$. Similarly, $\alpha$-synuclein oligomers intervene with organellar functions and interact with metal ions to cause oxidative stress ${ }^{14}$.

\section{(c) The Author(s) 2020}

(c) (i) Open Access This article is licensed under a Creative Commons Attribution 4.0 International License, which permits use, sharing, adaptation, distribution and reproduction cc) in any medium or format, as long as you give appropriate credit to the original author(s) and the source, provide a link to the Creative Commons license, and indicate if changes were made. The images or other third party material in this article are included in the article's Creative Commons license, unless indicated otherwise in a credit line to the material. If material is not included in the article's Creative Commons license and your intended use is not permitted by statutory regulation or exceeds the permitted use, you will need to obtain permission directly from the copyright holder. To view a copy of this license, visit http://creativecommons.org/licenses/by/4.0/. 
Mitochondrial abnormalities have also been associated with point mutations in Hsp70 chaperone that makes it aggregation prone, thereby, increasing iron and freeradical load ${ }^{15-17}$. Therefore, accelerated oxidation of biomolecules acts both as a product and causal for disease manifestation which is further exacerbated by failure of antioxidant system and loss of redox sensing capabilities of chaperone DJ-1 $1^{18,19}$.

The association of ROS with neuronal degeneration in PD is further substantiated by modeling the Parkinsonian phenotypes in animals through 1-methyl-4-phenyl-1,2,3,6tetrahydropyridine (MPTP), rotenone, 1,1'-dimethyl-4,4'bipyridinium dichloride (paraquat), or 6-hydroxydopamine (6-OHDA), those are known of elevate the oxygen freeradicals in the cell ${ }^{18}$. Substantial number of evidences proposes ROS to be a major contributor of dopaminergic neuronal loss in the PD brain. High ROS in SNpc neurons usually results from dopamine metabolism, low glutathione, and high levels of iron and calcium ${ }^{20}$. Dopamine is an unstable molecule that undergoes auto-oxidation or oxidative deamination through enzyme monoamine oxidases to generate peroxides ${ }^{21}$. In addition, presence of high concentrations of polyunsaturated fatty acids in the brain enhances their peroxidation and generation of toxic products which further causes degeneration of the cells harboring them or in vicinity ${ }^{22}$.

The contribution of oxidation in PD pathogenesis has also been supported through clinical studies reporting alterations in serum glutathione peroxidase and superoxide dismutase activities, together with high malondialdehyde (MDA) levels in PD patients ${ }^{23,24}$. Recent metanalysis have revealed significant increase in oxidative stress markers such as MDA and a substantial depletion of antioxidants such as catalase, GSH in the blood, and postmortem brain of PD patients compared to healthy controls $^{25}$. Therefore, regardless the clinical heterogeneity or underpinning genetics, the different forms of PD share a common etiology in mitochondrial dysfunction and oxidative stress.

Therefore, targeting cellular oxidative stress through antioxidants has been suggested as a potential therapeutic option against neurological disorders, particularly using natural plant extracts. Several studies have demonstrated effectiveness of different phytoderivatives in retracting disease pathogenesis, under various in vitro and in vivo models of PD ${ }^{26}$. In Drosophila MPTP probenecid model, mulberry fruit extracts were found to reduce nucleation of alpha-synuclein ${ }^{27}$. Similar results were obtained through Oleuropein derivatives from olive fruit extracts, which prevented $\alpha$-synuclein fibrillation and oligomer toxicity ${ }^{28}$. Extracts from Decalepis hamiltonii delayed early onset of PD symptoms, improved circadian rhythm and motor activity in PD model flies possessing A30P and A53T $\alpha$-synuclein mutation which are known to cause accelerated age dependent deterioration of circadian rhythm ${ }^{29}$. The extract also safeguarded the flies against paraquat induced oxidative stress and enhanced antioxidant defense ${ }^{29}$. Protection against paraquat treatment, locomotor impairment, shortened lifespan, and increased lipid peroxidation was observed in parkinRNAi flies treated with Persea americana peel extracts ${ }^{30}$. Administration of PINK1 $^{\text {B9 }}$ flies with Mucuna puriens extracts resulted in increased GSH amount and SOD activity, together with restoration of mitochondrial morphology in crop muscles, olfactory response, climbing behavior, extended lifespan, and delay in L-DOPA induced long term motor complication $^{31-33}$. However, the limited translation of these extracts into effective response in clinical trials, underlined further elucidation of potential candidates.

In the present study, we have reported a potent neuroprotective agent possessing robust antioxidant properties, isolated from Convolvulus pluricaulis, traditionally used as brain stimulator, memory enhancer and for mental debility. The presence of Scopoletin (Sp) is not limited to C. pluricaulis and characterized in many mental health promoting nootropic herbs such as Evolvulus alsinoides $^{34,35}$. Sp could efficiently restore redox balance in cells depleted for antioxidant properties or with dysfunctional mitochondria, mainly by enhancing the reducing potential of the cell through efficient recycling of GSH. The cytoprotective properties of $\mathrm{Sp}$ extended to decreased progression of degenerative phenotypes in Drosophila Parkinson model due to retention of redox balance in dopaminergic neurons and maintenance of their architecture. Sp could also increase the efficacy of L-DOPA, extensively used in palliative care of PD patients, by subsidence of its long term toxicity; highlighting its potential development as neuroprotective agent.

\section{Results \\ Scopoletin enhances antioxidant potential through replenishment of reduced glutathione}

The ability of $\mathrm{Sp}$ to combat intracellular oxidative stress was tested by inhibiting Catalase by 3 -amino, $1,2,4$ triazole (3-AT), followed by treatment with varying concentrations of the compound. The resultant elevation of oxidative radicals was found to be significantly reduced in cells further exposed to $250 \mu \mathrm{M}$ Sp (Fig. 1a, Supplementary Fig. S1b). The Sp treated cells recovered completely from stress induced cell death observed in case of 3-AT (Fig. 1b, Supplementary Fig. S1c, d), highlighting its role as a potential antioxidant and possible moiety associated with neuroprotective behavior of many nootropic herbs ${ }^{34}$. Indeed, Sp was able to restore cytosolic and mitochondrial redox balance in neuronal cell line SHSY-5Y subjected to 1-methyl-4-phenylpyridinium $\left(\mathrm{MPP}^{+}\right)$treatment which is known to cause mitochondria dysfunction and oxidative stress through complex I inhibition ${ }^{1}$ (Fig. 1c, d). Hence, 


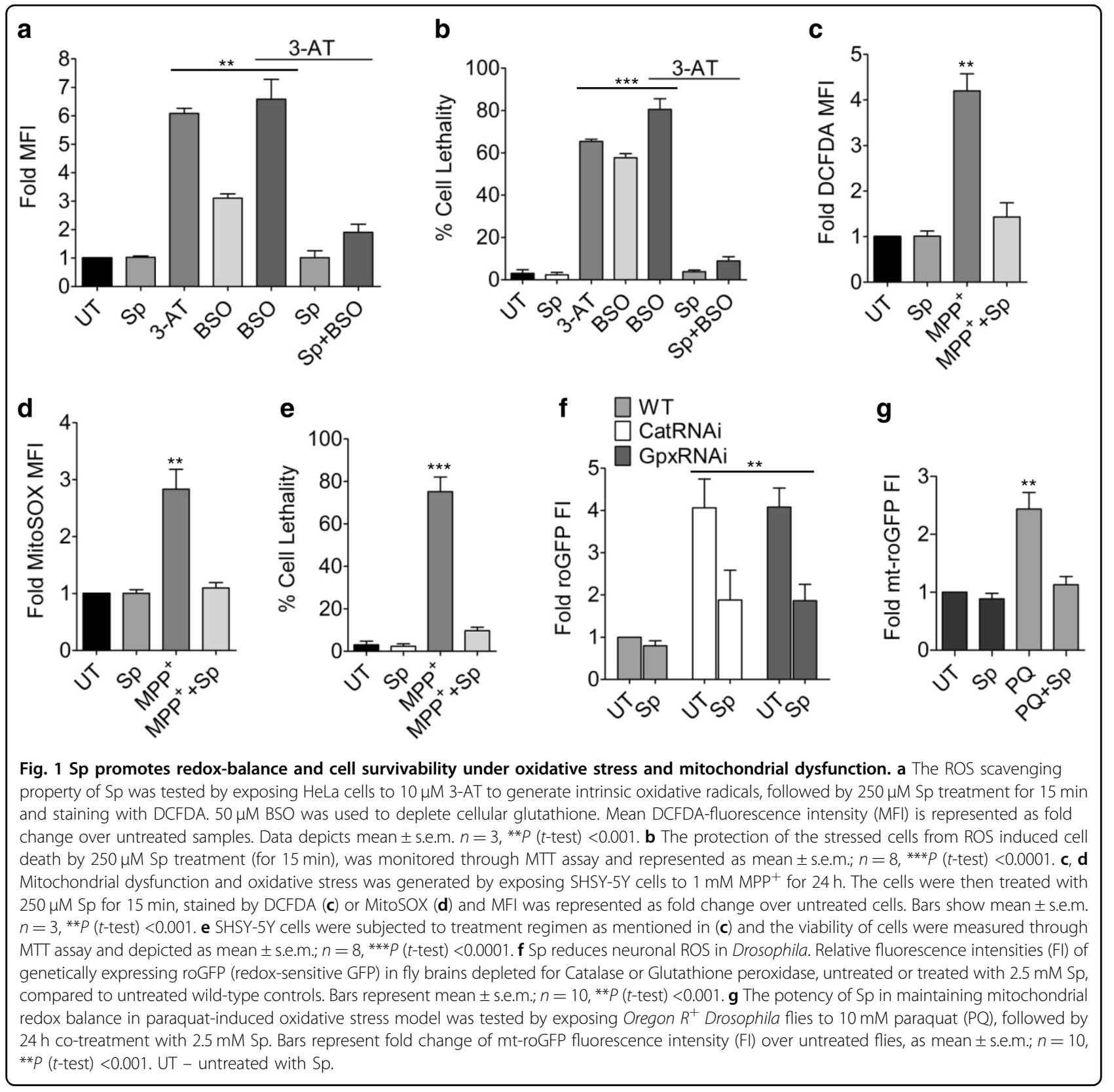

Sp treated neuronal cells showed enhanced survivability even after $\mathrm{MPP}^{+}$exposure (Fig. 1e). The Sp, by itself was found to be nontoxic at even higher concentrations (Supplementary Fig. S1a). The ROS scavenging property of Sp was also replicated in the Drosophila models knocked-down for essential antioxidant enzymes catalase and glutathione peroxidase, where neuronal cells of 15 days AEL flies genetically expressing a redox biosensor, roGFP showed reduced fluorescence under Sp treatment (Fig. 1f). A parallel phenotype was observed in paraquattreated fly model where Sp administration resulted in lower accumulation of oxygen radicals in mitochondria of brain neurons (Fig. 1g). The antioxidant property of $\mathrm{Sp}$ was however, found to be insensitive to depletion of glutathione by BSO, which generally causes loss of cellular antioxidant function and higher ROS (Fig. 1a). Sp treatment considerably reduced ROS and promoted higher cell survivability in BSO treated cells, in presence or absence of 3-AT, mainly by maintaining the availability of reduced glutathione (Fig. 1a, b). The cells treated with Sp showed slight enhancement in levels of GSH ( $\sim 1.2$ fold), together with minor alterations in GSSG levels, compared to untreated samples which exhibited higher GSSG content, thus indicating efficient recycling of GSSG to GSH (Fig. 2a). The idea was further validated by inhibiting glutathione synthesis by $\mathrm{BSO}$ where $\mathrm{Sp}$ administration 


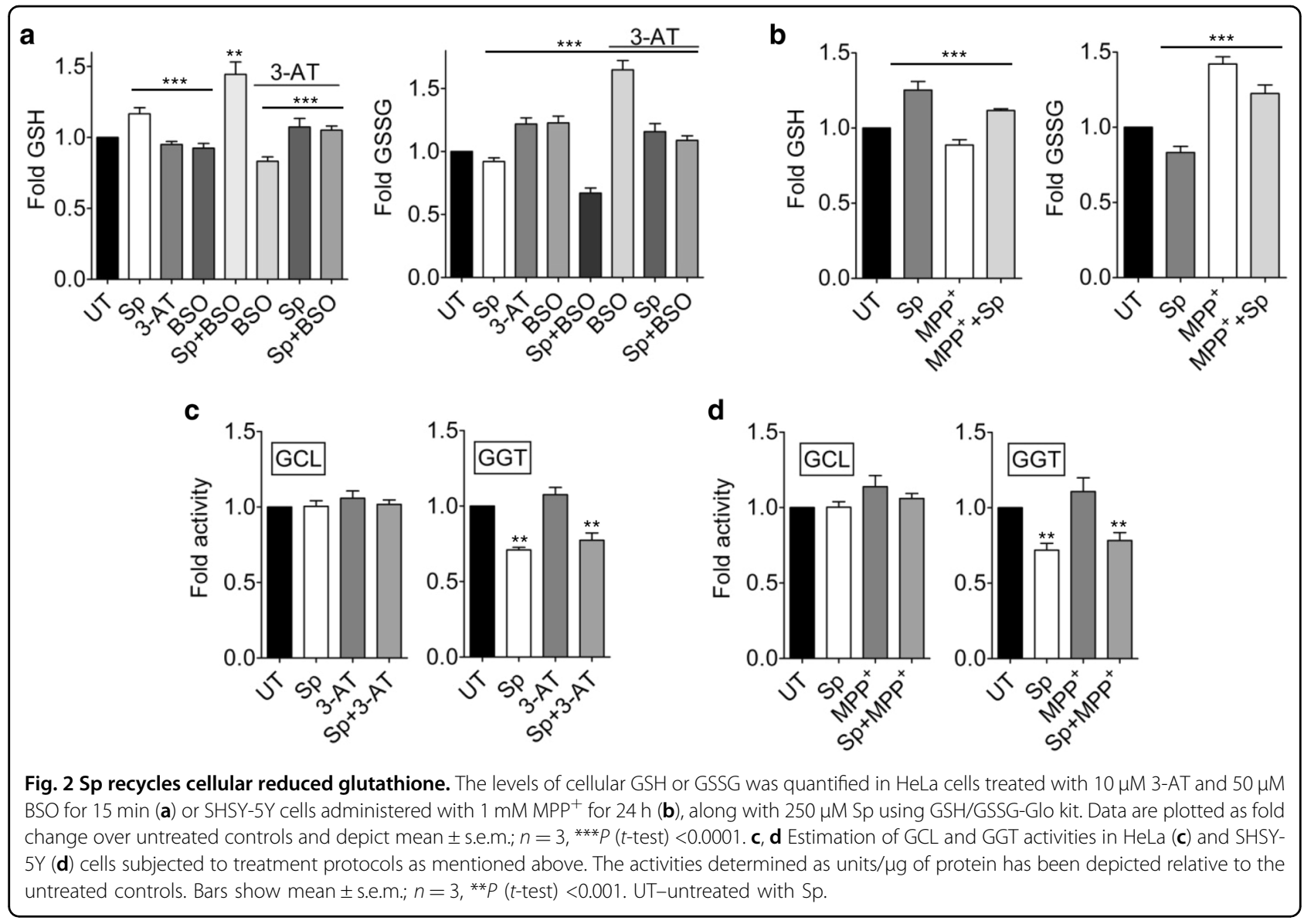

caused GSH to increase by $\sim 1.5$ fold with concomitant reduction in GSSG (Fig. 2a). The potency of Sp in maintaining the reducing equivalent was further reflected in superior GSH/GSSG ratio (approx. two fold) under BSO exposure in presence of 3-AT, and lower GSSG in BSO treated cells uninhibited for catalase (Fig. 2a). The capability of Sp to replenish reduced glutathione levels was further tested in neuronal cells exposed to $\mathrm{MPP}^{+}$, the toxic bioactivation product of MPTP known to induce Parkinsonian phenotype in animal models ${ }^{1}$. The levels of GSH was slightly reduced in $\mathrm{MPP}^{+}$-treated SHSY-5Y cells, which was further restored to baseline levels by $\mathrm{Sp}$, together with accompanying decrease in accumulated GSSG (Fig. 2b). The observed accumulation of GSH in Sp treated cells was probably due to reduced activity of $\gamma$ glutamyl transpeptidase (GGT), an enzyme responsible for glutathione catabolism ${ }^{36}$. Both HeLa and SHSY-5Y cells showed comparatively lower GGT activity upon Sp treatment, although the activities of glutamate-cysteine ligase were largely comparable (Fig. 2c, d). This indicates that antioxidant property of $\mathrm{Sp}$ encompasses replenishment of intracellular glutathione, probably through inhibition of its breakdown; thereby, making the cell more resistant to intrinsic or extraneous stressors.

\section{Amelioration of degenerative phenotypes in park ${ }^{13}$ dopaminergic neurons}

Oxidative stress is one of the major underlying causes of sporadic and familial forms of Parkinson's disease and mainly characterized by an imbalanced GSH/GSSG cycle, enhancing cellular susceptibility to oxidation ${ }^{37}$. The maintenance of glutathione cycle and survivability in neurotoxin exposed cells by $\mathrm{Sp}$, led us to test its plausibility in recovering the degenerative phenotypes of wellestablished Drosophila Parkinson mutant model park ${ }^{13}$. The dopaminergic neurons of park $^{13}$ and wild type control flies were marked with TH-Gal4 driven expression of UAS-GFP. Imaging of 20 days adult flies showed the neuronal architecture of Sp treated park ${ }^{13}$ flies similar to the wild type controls whereas their untreated counterparts showed shrinkage, aggregation, and complete disruption of dopaminergic neural networks (Fig. 3a). Expression of mt-roGFP in park $^{13}$ tyrosine hydroxylase positive neurons showed significantly reduced mitochondrial ROS accumulation under $\mathrm{Sp}$ treatment as compared to untreated ones, highlighting potential mitochondrial recovery and prevention of oxidation induced cell death in dopamine producing neurons (Fig. 3b). Recovery from the degenerative phenotypes and reduced 

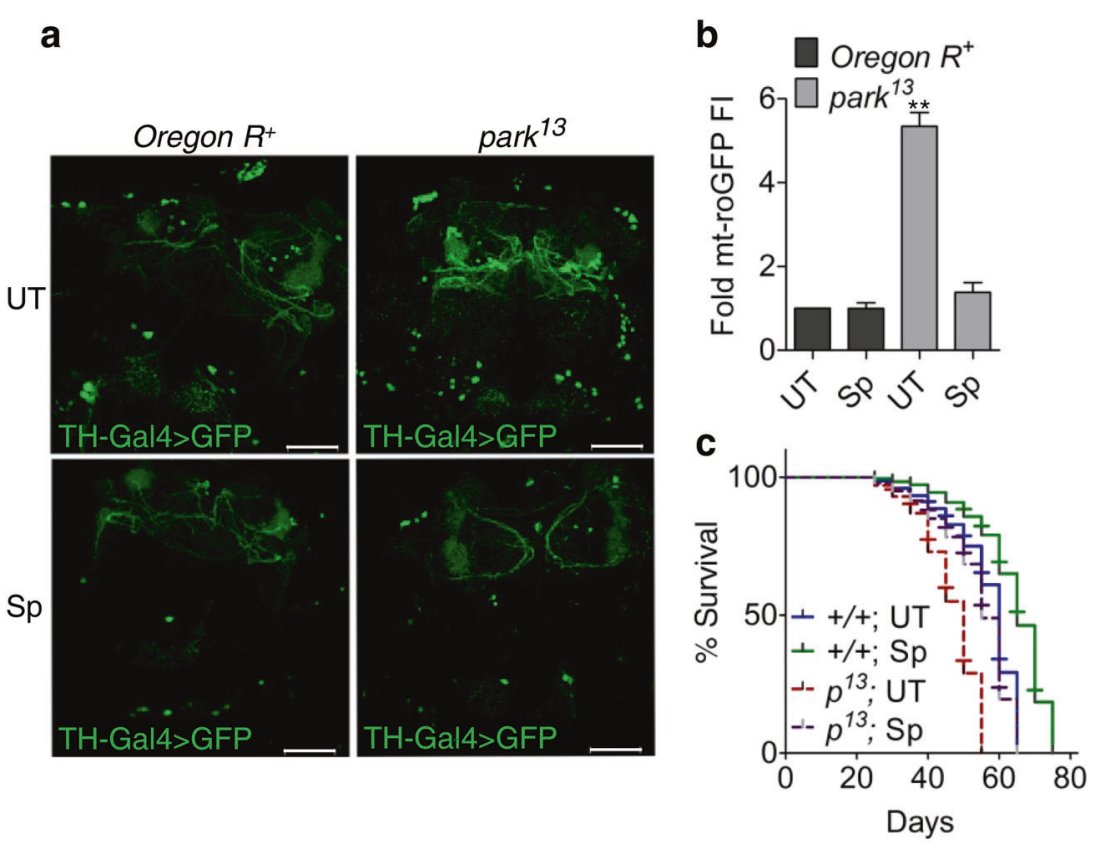

Fig. $3 \mathrm{Sp}$ promotes recovery of dopaminergic neurons in park flies. a Confocal images of Drosophila brains showing architecture of dopaminergic neurons labeled with GFP under wild type and mutant backgrounds, left untreated or treated with Sp. Scale bar: $50 \mu \mathrm{m}$. b The fluorescence intensity (FI) profile of park ${ }^{13}$ or wild-type Drosophila dopaminergic neurons expressing mt-roGFP under $2.5 \mathrm{mM}$ Sp treated or untreated conditions. Data is represented as fold difference over untreated wild-type control. Bars denote mean \pm s.e.m.; $n=3$, ${ }^{* *} P$ ( $t$-test $)<0.001$. c Kaplan Meier curve representing survivability pattern for wild type and park ${ }^{13}\left(p^{13}\right)$ flies untreated or treated continuously with 2.5 mM Sp; ${ }^{* * *} P$ (Mantel-Cox test) $<0.0001$. UT - untreated with Sp.

oxidative stress therefore, contributed to better survivability of the Sp-park ${ }^{13}$ flies, which was comparable to Oregon $R^{+}$(Fig. 3c). Sp treated wild-type flies though presented a better life span than untreated controls, did not show any significant difference in their oxidative profile and neural organization; nor were found susceptible to extraneous stressors, highlighting that Sp exposure did not compromise organismal response to electrophiles (Fig. 3c, Supplementary Fig. S1e).

\section{Prevention of oxidative damage and maintenance of mitochondrial function}

The neuroprotective behavior displayed by Sp intrigued a parallel study in the Drosophila eye since it displays detectable phenotypes in response to neurophysiological disruptions such as progressive loss of ommatidia accompanied with disrupted rhabdomere arrangement ${ }^{38}$. Immunohistochemical analysis of eye imaginal disks from $120 \mathrm{~h}$ AEL third instar larvae, showed better rhabdomere arrangement in $\mathrm{Sp}$ treated park $^{13}$ flies as compared to untreated ones (Fig. 4a). Sp treatment resulted in presence of $\sim 270$ rhabdomeres close to mean wild type number of 290, an improvement over $\sim 200$ rhabdomeres observed in the mutants (Fig. 4b). The cytoprotective role of Sp was further evaluated through determination of mitochondrial function and amount of intracellular lipid peroxides quantified by measuring the levels of their decomposed carbonyl product malondialdehyde; the two components most vulnerable to oxidative damage due to increased mass of polyunsaturated fatty acids and respiratory activity in neurons. The park ${ }^{13}$ flies showed higher amounts of lipid peroxides which were considerably reduced upon Sp exposure (Fig. 4c). Similarly, Sp treatment resulted in maintained mitochondrial complex I activity which is found to be disrupted in PD patients and Parkinsonian models (Fig. 4d). As a result, the Sp-park ${ }^{13}$ flies presented healthier mitochondria sustaining higher membrane potential, as suggested by increased TMRE staining (Fig. $4 \mathrm{e}$ ) and higher accumulation of mitochondrial mass, probably due to prevention of ROS-induced organellar damage (Fig. 4f). The Sp-flies, in-spite of possessing increased mitochondrial content, demonstrated reduced production of mitochondrial superoxides (Fig. 4g); thereby, promoting the observed neuronal recovery.

\section{Improved motor co-ordination in Scopoletin treated park ${ }^{13}$ flies}

To test whether the improvement in neuronal abnormalities translated to rehabilitation of aberrant movement phenotypes including motion slowness, difficulties with gait and balance, we utilized the negative geotactic and phototactic behavior of Drosophila flies. 


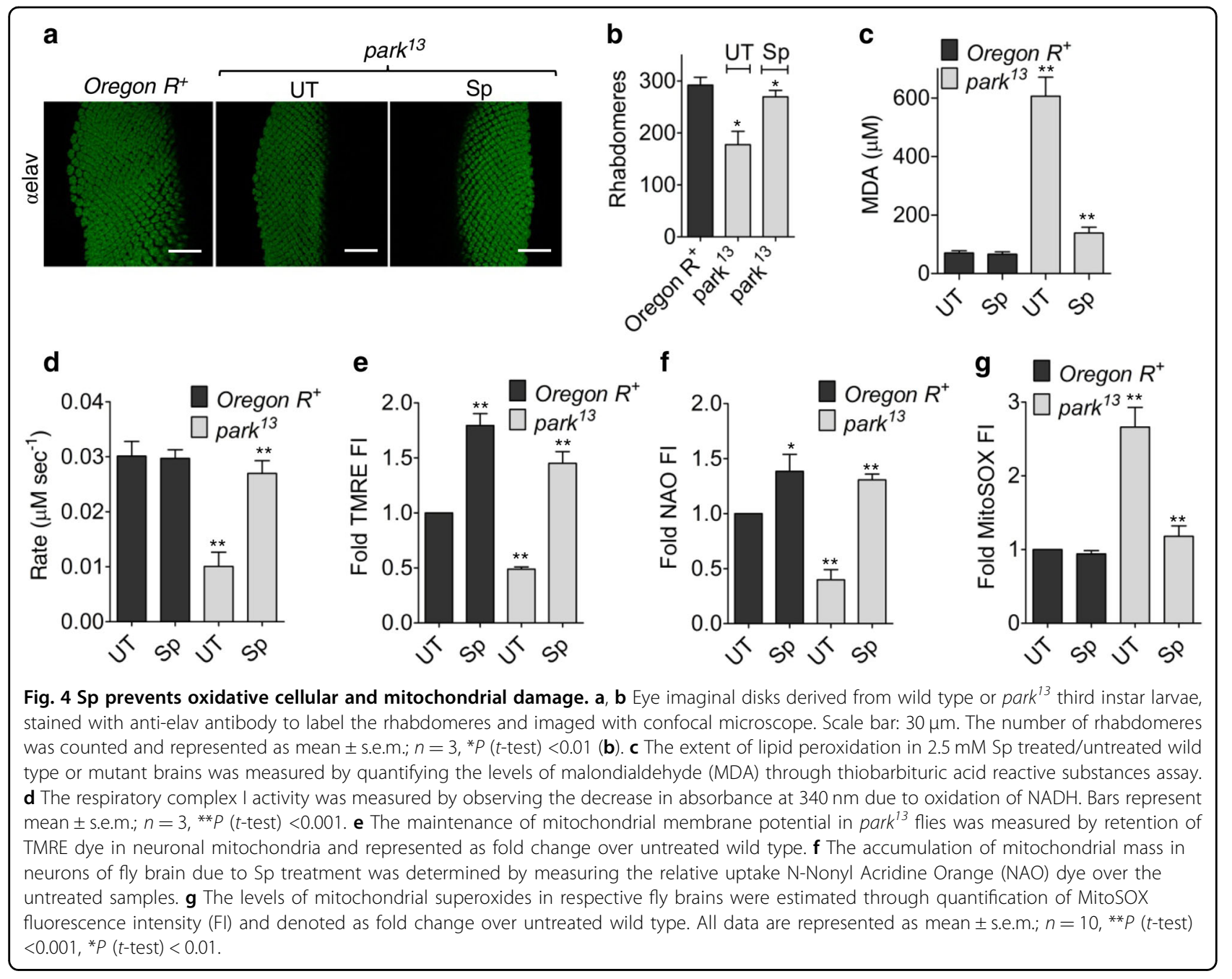

The wild type flies, irrespective of their treated conditions showed strong negative geotaxis through a vertical column. In contrast, the neuromotor dysfunction in park $^{13}$ flies reflected locomotion defects where the mutant flies showed $>50 \%$ compromised climbing activity compared to wild type. Sp treatment resulted in appreciable recuperation of the locomotor activity close to normal levels (Fig. 5a). Similarly, disoriented movement in park $^{13}$ due to absence of photosensing capability was reasonably restored in $\mathrm{Sp}$ treated flies. The flies showed positive phototactic orientation quite similar to wild type pattern (Fig. 5b); hence, demonstrating efficaciousness of Sp in managing motor control abnormalities.

\section{Scopoletin alleviates toxicity associated with long term exposure to L-DOPA}

L-DOPA has been the mainstay and most efficacious in management of PD phenotypes. However, its administration has been plagued by absence of long term benefits, oxidative toxicity being one of the major reasons thus, freezing the patients mid-sentence ${ }^{39,40}$. Although continuous treatment of park $^{13}$ flies with L-DOPA reduced the inherent oxidative stress consistent with previous results, the flies still showed considerably higher levels of oxidative radicals (Fig. 5c). A similar observation was seen in L-DOPA treated wild type flies. The increased levels of L-DOPA dependent ROS was significantly reduced upon Sp exposure, both in wild type and park ${ }^{13}$ flies (Fig. 5c). The alleviation of L-DOPA toxicity by $\mathrm{Sp}$ was reflective of non-stasis in the recovery of motor abilities in park $^{13}$ flies and the motility of the flies for extended period was comparable to the controls (Fig. 5e). L-DOPA alone contributed to strong recovery of motion phenotypes during initial phase, however, its effect lagged in aged flies which showed movement defects similar to untreated park $^{13}$ mutants (Fig. 5e), probably due to dyskinesia usually associated with protracted L-DOPA exposure. The combination did not show any apparent deleterious effect in wild type controls (Fig. 5d), indicative of their potential usage in PD care. 


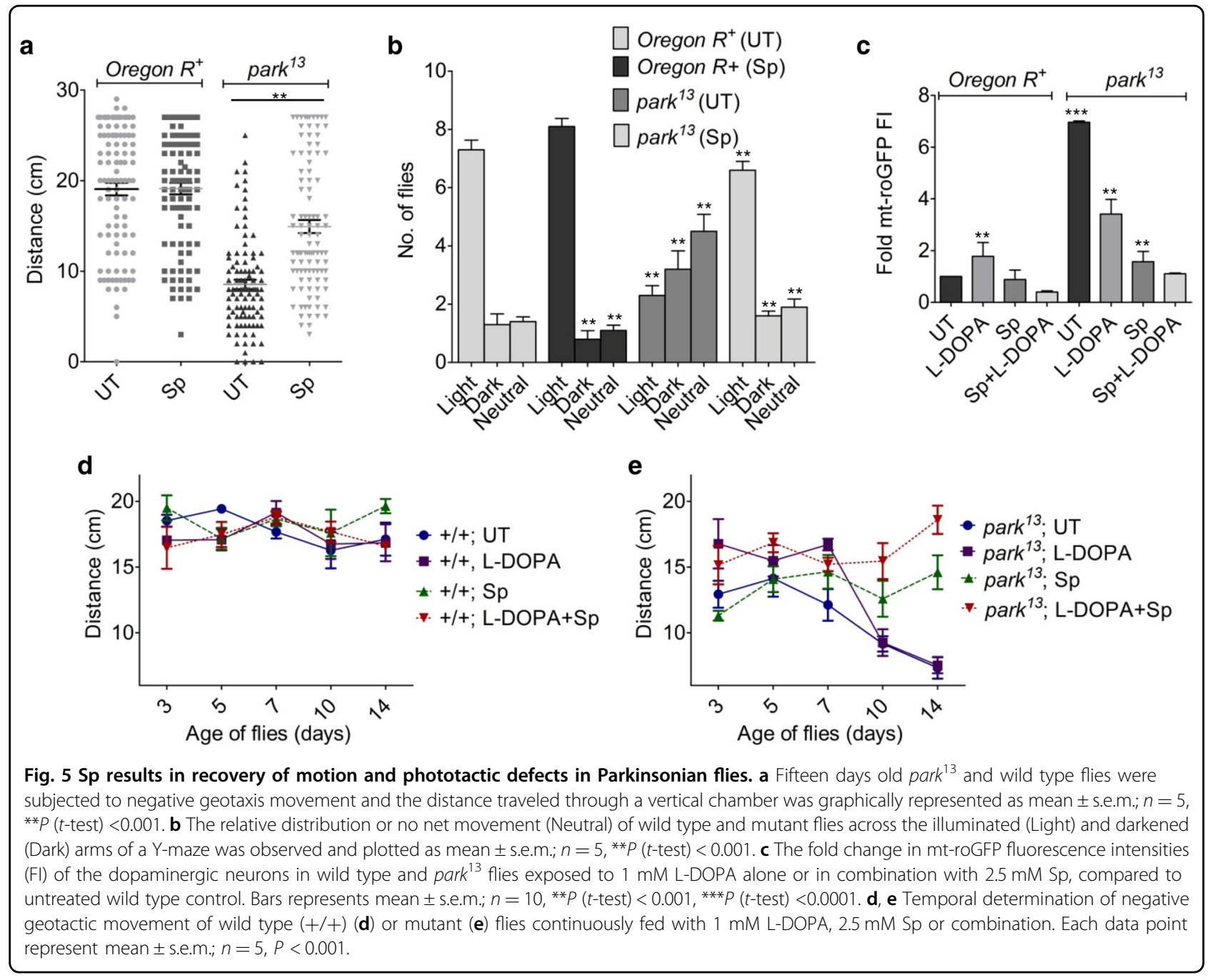

\section{Discussion}

The vulnerability of dopaminergic neurons to minor perturbations in redox equilibrium is enhanced by the oxidative by-products of dopamine metabolism, resulting in their selective loss in Parkinson's disease. In the nigrostriatal tract system, dopamine undergoes autooxidation or oxidative deamination by monoamine oxidase- $B$, both of which aggravates with age, leading to increased production of peroxides and hydroxyl radicals $^{41}$. In addition, age dependent accretion of dysfunctional mitochondria elevates stress level, which is exhibited by high levels oxidized products such as protein carbonyls and lipid peroxides ${ }^{42}$. The failure of the antioxidant system to negate the pathological rise in the oxidative species is exacerbated by reduction in levels of the major cellular nucleophile, GSH due to their covalent modification by dopamine quinone species ${ }^{20,43-45}$. Therefore, complementing the resistance of redox equilibrium to oxidative shift by supplementing the GSH levels has been considered to be one of the plausible approaches to contain the progressiveness of the disorder ${ }^{46}$.

A large number of antioxidant based strategies focusing on ascorbic acid, deferoxamine, cucurmin etc has been investigated for management of the PD symptoms but without any translatable benefits ${ }^{47,48}$; thus requiring elucidation of better alternatives with robust antioxidant capabilities to promote neuronal health in PD. Natural derivatives have been increasingly appreciated for their potency in prevention and management of neurodegenerative diseases $^{35,49,50}$. Convolvulus pluricaulis extract is one of the most widely used neuropharmacological agent that significantly improved learning and memory in experimental models ${ }^{34,51,52}$. It has been employed in treatment of dementia, phobias and insomnia, and successfully tried in PD cases ${ }^{53}$. C. pluricaulis also has been found to ameliorate tau induced neurotoxicity in Alzhei-

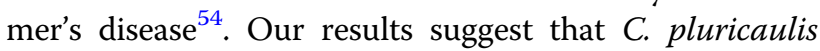
majorly derives its neuroprotective properties through a robust antioxidant $\mathrm{Sp}$. 
Sp was able to restore cellular redox homeostasis under induced oxidative stress in mammalian cells and in vivo model, Drosophila where it ameliorated catalase and glutathione peroxidase deficiency. Further exposure of the Parkinsonian flies to Sp resulted in diseased flies to display more number of viable non-stressed dopaminergic neurons than the non-treated ones. Higher mitochondrial metabolism in the neurons result in generation of Complex I dependent hydrogen peroxide and neuronal membranes, rich in polyunsaturated fatty acids, are particularly vulnerable to oxidative stress due to their possession of unsaturated double bonds. Peroxidation of lipids disturbs membrane organization, causing functional loss of proteins and DNA, and are found elevated in PD patients where lipid peroxidation adducts contribute to early demise of the SNpc neurons. Mitochondria being the source and target of lipid peroxides accelerate the process $^{4,8,20}$. Sp presented commendable recovery of the mutant flies from accumulated lipid peroxides and also induced parallel recovery of mitochondrial health; thus translating into better neuronal architecture observed in treated park flies. The potent neuro-protective ability of Sp was mainly derived from its ability to maintain glutathione levels in its oxidizable form, possibly by preventing its catabolism, though the exact chemical mechanism needs to be elucidated through further studies. The idea is supported by maintenance of functional GSH/GSSG levels by Sp in BSO and neurotoxin treated cells as compared to untreated ones which showed a significant decline in the antioxidant ratio probably, due to enhanced formation of glutathione-quinones ${ }^{43}$; hence, allowing Sp to effectively circumvent the ROS induced toxicity associated with the prevailing therapies.

The considerable recovery of the dopaminergic neuronal clusters in the park flies ensued better negative geotactic behavior in the flies and prolonged their life span, suggesting improvement in motor coordination dysfunction associated with PD. The flies solely administered with L-DOPA performed better during initial exposure after which their progress declined, consistent with the reported pattern of "wearing-off" in L-DOPA administered PD patients $^{39,55}$. However, Sp-L-DOPA combination showed consistent negative geotaxis even during the "wearing-off" phase, representing substantial alleviation of L-DOPA toxicity and promotion of enhanced mitochondrial and cellular health in dopaminergic neurons.

Notwithstanding the strides achieved in genetic manipulation and regenerative technologies, L-DOPA or compounds related to dopamine metabolism such as MAO-B inhibitors remain as the major modes of palliative care for PD patients ${ }^{39,56}$. However, these regimens have been plagued with long term drug toxicity leading to retarded neutrite growth, mitochondrial dysfunction, and oxidative stress $^{18}$. Enhancement of the intracellular reducing equivalents such as glutathione, to increase the cellular competence to electrophiles holds promise to impede the progression of the disorder and had been clinically effective in PD patients. Standalone or combinatorial regimens designed around potent bio-absorptive reductants, elevating the efficiency of palliative treatment by decreasing or delaying disease progression, will be a welcome step for further trials and those affected by the disease.

\section{Material and methods Cell culture}

HeLa (ATCC Number: CCL-2) and SHSY-5Y (ATCC Number: CRL-2266) cells, STR profiled and free from any contamination, were sourced from National Center for Cell Science repository. The cells were cultured in Eagle's minimum essential medium (ThermoFisher Scientific) or DMEM F-12 (ThermoFisher Scientific) respectively, supplemented with $10 \%$ Fetal Bovine Serum (Gibco) and 1\% penicillin-streptomycin (ThermoFisher Scientific), in a humidified incubator maintaining $5 \% \mathrm{CO}_{2}$ at $37^{\circ} \mathrm{C}$. All assays were performed in cells maintained at $\sim 60 \%$ confluency.

\section{Fly strains}

UAS-GFP (Bloomington Stock \#6874), TH-Gal4 (Bloomington Stock \#8848), CatalaseRNAi (Bloomington Stock \#34020) and PHGPx (Bloomington Stock \#33939), were obtained from Bloomington stock center, Indiana, along with park $^{13}$ (Bloomington Stock \#79210) as the Parkinsonian disease model. Oregon $R^{+}$was used as the wild type control line for all experiments. The stocks were maintained in $24^{\circ} \mathrm{C}$ incubator. The genetic combinations used in the assays were generated by standard genetic crosses.

\section{ROS measurement}

Oxidative stress was induced by exposing $1.5 \times 10^{5}$ HeLa cells seeded overnight, to $10 \mu \mathrm{M} 3-\mathrm{AT}$ for $15 \mathrm{~min}$ at $37^{\circ} \mathrm{C}$; followed by treatment with $250 \mu \mathrm{M}$ of the Sp for $15 \mathrm{~min}$. Cellular glutathione levels were depleted by coexposing the cells to $50 \mu \mathrm{M}$ BSO for $15 \mathrm{~min} .1 .2 \times 10^{5}$ SHSY-5Y cells were subjected to similar treatment as above except $1 \mathrm{mM} \mathrm{MPP}^{+}$(for $24 \mathrm{~h}$ ) was used to induce ROS. Post-treatment, the cells were washed with $1 \mathrm{X}$ phosphate-buffered saline and stained with $15 \mu \mathrm{M}$ DCFDA-H2 or $5 \mu \mathrm{M}$ MitoSOX for $15 \mathrm{~min}$ at $37^{\circ} \mathrm{C}$. The estimation of total cellular GSH and GSSG levels was performed through GSH/GSSG-Glo ${ }^{\mathrm{TM}}$ Assay Kit (Promega) as per manufacturer's instructions. GGT and GCL activity was determined through GGT-Activity Colorimetric Assay Kit (Merck Sigma-Aldrich) and GCL Assay Kit (Real-Gene Labs) respectively, following the protocol detailed by the manufacturer. The observed activities were normalized over the total protein content estimated through Quick Start ${ }^{\mathrm{TM}}$ Bradford reagent (Bio-Rad). 
Fluorescence/luminescence/absorbance intensities at appropriate wavelengths were quantified in Synergy H1 microplate reader (version 2.09.1). In case of Drosophila, neuronal redox balance was altered by driving CatalaseRNAi or PHGpxRNAi, along with roGFP by elav-Gal4 to deplete the respective antioxidant enzymes and express the redox biosensor. The first instar larvae taken $24 \mathrm{~h}$ AEL (after egg laying), was orally administered with $2.5 \mathrm{mM} \mathrm{Sp}$ and fluorescence measurement in brains of eclosed adults were performed as mentioned above. To test the effect of $\mathrm{Sp}$ in paraquat-induced oxidative stress model, seven days old wild type flies, constitutively expressing mitochondrial $(\mathrm{mt})$-roGFP, were exposed to $10 \mathrm{mM}$ paraquat for $24 \mathrm{~h}$ and then co-treated with $2.5 \mathrm{mM} \mathrm{Sp}$ for $24 \mathrm{~h}$, prior to roGFP fluorescence estimation. In case of park $^{13}$, mtroGFP was expressed in dopaminergic neurons by $\mathrm{TH}-$ Gal4 for estimation of redox state in twenty days old adult flies either individually treated with $2.5 \mathrm{mM}$ Sp or cotreated with $1 \mathrm{mM}$ L-DOPA from 10 days AEL. The concentrations of Sp and L-DOPA were kept similar for all experiments unless otherwise mentioned. Each replicate constituted of twenty flies. Equivalent numbers of age-matched unsegregated flies were used for all experiments except survival assay.

\section{Cellular viability}

Optimization of $\mathrm{Sp}$ concentration and its cytotoxicity parameters were established by treating of $1.5 \times 10^{5} \mathrm{HeLa}$ or $1.2 \times 10^{5}$ SHSY-5Y cells with serial concentrations of the Sp $(100 \mu \mathrm{M}-1 \mathrm{mM})$ for $15 \mathrm{~min}$ or conducting a timecourse $(5 \mathrm{~min}-48 \mathrm{~h}$ ) using $250 \mu \mathrm{M} \mathrm{Sp}$, followed by MTT assay as per standard protocol (Thermo Fisher Scientific). The absorbance was taken at $540 \mathrm{~nm}$ in Synergy H1 microplate reader (version 2.09.1). The rescue of cell viability by $\mathrm{Sp}$ was measured by following a similar treatment regimen as described above for ROS profiling of the cells. Recovery from ROS-induced toxicity was tested through MTT assay, in HeLa and SHSY-5Y cells pre-exposed to $10 \mu \mathrm{M} 3$-AT for $15 \mathrm{~min}$ or $1 \mathrm{mM} \mathrm{MPP}{ }^{+}$for $24 \mathrm{~h}$ respectively, followed by $250 \mu \mathrm{M} 15 \mathrm{~min}$ Sp treatment.

\section{Confocal imaging}

Tyrosine hydroxylase (TH)-Gal4 driven UAS-GFP lines, labeling the neurons with active dopamine synthesis pathway, were crossed with park $^{13}$ flies and progenies were exposed to Sp from 10 days AEL. Twenty days old adult brains from the resultant $\mathrm{F} 1$ progeny carrying the genotype $T H>$ Gal4; $_{\text {park }}{ }^{13}$, were imaged under Sp treated and untreated conditions to assess the integrity of dopaminergic neurons. Rhabdomere arrangement was visualized in third instar (120 h AEL, Sp fed for $48 \mathrm{~h}$ ) larval eye imaginal disc by labeling with anti-elav antibody (9F8A9, DSHB). Image analysis was performed through Zeiss 510 META confocal microscope and processed with LSM image browser. Rhabdomere quantification was done through ImageJ. Images were minimally processed for brightnesscontrast adjustments using Adobe Photoshop 7.0.

\section{Assessment of mitochondrial function}

Ten numbers of anesthetized twenty day old adult flies were dissected in cold Drosophila Schneider's Medium (DSM). The dissected brains were incubated in $100 \mathrm{nM}$ TMRE solution (Thermo Fisher Scientific) in DSM for $10 \mathrm{~min}$ and relative fluorescence intensity was quantified by top excite-read method through Synergy H1 reader. For estimation of mitochondrial mass, the fly brains were stained with $20 \mu \mathrm{M}$ N-Nonyl Acridine Orange for $15 \mathrm{~min}$ and fluorescence intensity was determined as above. Quantification of mitochondrial superoxide was similarly executed except that the samples were stained with $5 \mu \mathrm{M}$ MitoSox ${ }^{\mathrm{TM}}$ Red (Thermo Fisher Scientific). Activity of complex I was quantified based on the protocol described by Spinazzi et al. ${ }^{57}$ Mitochondria, isolated using mitochondria isolation kit (Sigma), were incubated in phosphate buffer containing $1 \%$ DOC and $2 \mathrm{mM}$ sodium azide. After $15 \mathrm{~min}$, mitochondria were incubated with $1 \mathrm{mM}$ $\mathrm{NADH}$. The reaction was initiated with the addition of $60 \mu \mathrm{M}$ ubiquinone and the decrease in absorbance was recorded at $340 \mathrm{~nm}$ for $2 \mathrm{~min}$.

\section{Lipid peroxidation}

The brains of 20 days old wild type and mutant flies, Sp treated or kept untreated as mentioned above, were dissected and homogenized in DSM. Then, $1 \%$ thiobarbituric acid was added to the clarified homogenate and incubated at $90^{\circ} \mathrm{C}$ for $1 \mathrm{~h}$, followed by addition of $250 \mu \mathrm{L}$ of $\mathrm{n}$ butanol. The solution was centrifuged at $16,000 \times g$ for $2 \mathrm{~min}$ and the supernatant was used to measure absorbance at $530 \mathrm{~nm}$.

\section{Behavioral assays}

Phototactic Assay was conducted to evaluate optic nerve degeneration, employing Y-shaped glass maze with lightened, dark and light neutral ends. Ten flies per replicate were introduced in the neutral end and their migration into each branch was observed. Assessment of neuromuscular coordination was performed by evaluating the ability of the flies to ascend a vertical graduated plastic cylinder. Each set consisted of five flies kept at the bottom of the tube. The tube was gently shaken and the final distance traveled within $10 \mathrm{~s}$ was recorded. 20 days old flies, $\mathrm{Sp}$ and/or L-DOPA fed from 10 days AEL, was used in all the experiments.

\section{Survival assay}

Measurement of life span in wild type and park ${ }^{13}$ flies was essentially performed as per established protocols ${ }^{58}$. Briefly, 100 age-matched male and female flies were 
segregated in separate vials and transferred to fresh vials during the experimental period every five days. Dead flies were recorded at the time of each transfer process. Flies exiting the experiment were censored and not recorded as dead. Data was subjected to Kaplan Meier analysis in Graphpad Prism.

\section{Statistical analysis}

The sample size possessing sufficient statistical power for each experiment is provided in the figures or mentioned under respective sub-headings in the methods section. Data are representative of minimum three independent experiments and the number of biological replicates is quoted in the figure legends. Mean and standard error was calculated for all quantitative experiments. Statistical significance was determined using twotailed Student's $t$ test through GraphPad Prism5 software unless otherwise mentioned. The $P$ values have been represented by comparing the data pairwise with wild type untreated controls and are mentioned in figure legends. Statistical significance was specified as ${ }^{*} P<0.01$, ${ }^{* *} P<$ 0.001 , or ${ }^{* * *} P<0.0001$.

\section{Acknowledgements}

We thank Prof. S. C. Lakhotia, Prof. J. K. Roy and members of Cytogenetics lab, $\mathrm{BHU}$ for providing Drosophila strains and sharing their lab facilities. We also thank BHU Confocal facility for imaging experiments and Prof. S. K. Trigun lab for microplate reader. This work is supported by Young Scientist grant by Indian National Science Academy (INSA/SP/YSP/152/2018/232), DST-FIST Program (No. SR/FST/LS-II/2017/107) and UGC CAS support (No. F.5-10/2018/ CAS-VI (SAP-II)). The authors also acknowledge financial support from UGC-CAS doctoral fellowship (to P.P.), DST-INSPIRE fellowship (to O.M.) and CSIR-Junior research fellowship (to A.B.).

\section{Conflict of interest}

The authors declare that they have no conflict of interest.

\section{Publisher's note}

Springer Nature remains neutral with regard to jurisdictional claims in published maps and institutional affiliations.

Supplementary Information accompanies this paper at (https://doi.org/ 10.1038/s41419-020-02942-8).

Received: 16 April 2020 Revised: 23 August 2020 Accepted: 27 August 2020 Published online: 10 September 2020

\section{References}

1. Dauer, W. \& Przedborski, S. Parkinson's disease: mechanisms and models. Neuron 39, 889-909 (2003).

2. Spillantini, M. G. et al. Alpha-synuclein in Lewy bodies. Nature $\mathbf{3 8 8}$, 839-840 (1997).

3. Stefanis, L. alpha-Synuclein in Parkinson's disease. Cold Spring Harb. Perspect. Med 2, a009399 (2012).

4. Poewe, W. et al. Parkinson disease. Nat. Rev. Dis. Prim. 3, 17013 (2017).

5. Trinh, J. \& Farrer, M. Advances in the genetics of Parkinson disease. Nat. Rev. Neurol. 9, 445-454 (2013).

6. Lill, C. M. Genetics of Parkinson's disease. Mol. Cell Probes 30, 386-396 (2016).

7. Blauwendraat, C., Nalls, M. A. \& Singleton, A. B. The genetic architecture of Parkinson's disease. Lancet Neurol. 19, 170-178 (2020).
8. Blesa, J., Trigo-Damas, I., Quiroga-Varela, A. \& Jackson-Lewis, V. R. Oxidative stress and Parkinson's disease. Front Neuroanat. 9, 91 (2015).

9. Hemmati-Dinarvand, M. et al. Oxidative stress and Parkinson's disease: conflict of oxidant-antioxidant systems. Neurosci. Lett. 709, 134296 (2019).

10. Park, J. S., Davis, R. L. \& Sue, C. M. Mitochondrial dysfunction in parkinson's disease: new mechanistic insights and therapeutic perspectives. Curr. Neurol. Neurosci. Rep. 18, 21 (2018).

11. Jones, N. PINK1 targets dysfunctional mitochondria for autophagy in Parkinson disease. Nat. Rev. Neurol. 6, 181 (2010).

12. Pickrell, A. M. \& Youle, R. J. The roles of PINK1, parkin, and mitochondrial fidelity in Parkinson's disease. Neuron 85, 257-273 (2015).

13. Jin, S. M. \& Youle, R. J. PINK1- and Parkin-mediated mitophagy at a glance. J. Cell Sci. 125, 795-799 (2012)

14. Wood-Kaczmar, A., Gandhi, S. \& Wood, N. W. Understanding the molecular causes of Parkinson's disease. Trends Mol. Med. 12, 521-528 (2006).

15. Burbulla, L. F. et al. Dissecting the role of the mitochondrial chaperone mortalin in Parkinson's disease: functional impact of disease-related variants on mitochondrial homeostasis. Hum. Mol. Genet. 19, 4437-4452 (2010).

16. Isaya, G. Mitochondrial iron-sulfur cluster dysfunction in neurodegenerative disease. Front. Pharm. 5, 29 (2014).

17. Goswami, A. V., Samaddar, M., Sinha, D., Purushotham, J. \& D'Silva, P. Enhanced J-protein interaction and compromised protein stability of mtHsp70 variants lead to mitochondrial dysfunction in Parkinson's disease. Hum. Mol. Genet. 21, 3317-3332 (2012)

18. Dias, V., Junn, E. \& Mouradian, M. M. The role of oxidative stress in Parkinson's disease. J. Parkinsons Dis. 3, 461-491 (2013).

19. Irrcher, I. et al. Loss of the Parkinson's disease-linked gene DJ-1 perturbs mitochondrial dynamics. Hum. Mol. Genet. 19, 3734-3746 (2010).

20. Jenner, P. \& Olanow, C. W. The pathogenesis of cell death in Parkinson's disease. Neurology 66, S24-S36 (2006).

21. Munoz, P., Huenchuguala, S., Paris, I. \& Segura-Aguilar, J. Dopamine oxidation and autophagy. Parkinsons Dis. 2012, 920953 (2012).

22. Liu, X., Yamada, N., Maruyama, W. \& Osawa, T. Formation of dopamine adducts derived from brain polyunsaturated fatty acids: mechanism for Parkinson disease. J. Biol. Chem. 283, 34887-34895 (2008).

23. Riederer, P., Konradi, C. \& Hebenstreit, G. [Neurochemical perspectives of the function of monoamine oxidases]. Psychiatr. Prax. 16(Suppl 1), 7-10 (1989).

24. Yoritaka, A. et al. Immunohistochemical detection of 4-hydroxynonenal protein adducts in Parkinson disease. Proc. Natl Acad. Sci. USA 93, 2696-2701 (1996).

25. Wei, Z., Li, X., Liu, Q. \& Cheng, Y. Oxidative stress in Parkinson's disease: a systematic review and meta-analysis. Front Mol. Neurosci. 11, 236 (2018).

26. Pohl, F. \& Kong Thoo Lin, P. The potential use of plant natural products and plant extracts with antioxidant properties for the prevention/treatment of neurodegenerative diseases: in vitro, in vivo and clinical trials. Molecules 23, 3283 (2018).

27. Gu, P. S., Moon, M., Choi, J. G. \& Oh, M. S. Mulberry fruit ameliorates Parkinson'sdisease-related pathology by reducing alpha-synuclein and ubiquitin levels in a 1-methyl-4-phenyl-1,2,3,6-tetrahydropyridine/probenecid model. J. Nutr. Biochem. 39, 15-21 (2017)

28. Mohammad-Beigi, $\mathrm{H}$. et al. Oleuropein derivatives from olive fruit extracts reduce alpha-synuclein fibrillation and oligomer toxicity. J. Biol. Chem. 294 4215-4232 (2019)

29. Jahromi, S. R., Haddadi, M., Shivanandappa, T. \& Ramesh, S. R. Attenuation of neuromotor deficits by natural antioxidants of Decalepis hamiltonii in transgenic Drosophila model of Parkinson's disease. Neuroscience 293, 136-150 (2015).

30. Ortega-Arellano, H. F., Jimenez-Del-Rio, M. \& Velez-Pardo, C. Neuroprotective effects of methanolic extract of avocado Persea americana (var. Colinred) peel on paraquat-induced locomotor impairment, lipid peroxidation and shortage of life span in transgenic knockdown Parkin Drosophila melanogaster. Neurochem. Res. 44, 1986-1998 (2019).

31. Baroli, B. et al. Evaluation of oxidative stress mechanisms and the effects of phytotherapic extracts on Parkinson's disease Drosophila PINK1(B9) model. FASEB J. 33, 11028-11034 (2019).

32. Solari, P. et al. The imbalance of serotonergic circuitry impairing the crop supercontractile muscle activity and the mitochondrial morphology of PD PINK1(B9)Drosophila melanogaster are rescued by Mucuna pruriens. J. Insect Physiol. 111, 32-40 (2018).

33. Poddighe, S. et al. Mucuna pruriens (Velvet bean) rescues motor, olfactory, mitochondrial and synaptic impairment in PINK1B9 Drosophila melanogaster genetic model of Parkinson's disease. PLoS One 9, e110802 (2014). 
34. Sethiya, N. K., Nahata, A., Singh, P. K. \& Mishra, S. H. Neuropharmacological evaluation on four traditional herbs used as nervine tonic and commonly available as Shankhpushpi in India. J. Ayurveda Integr. Med. 10, 25-31 (2019).

35. Cervenka, F. et al. Evaluation of natural substances from Evolvulus alsinoides L. with the purpose of determining their antioxidant potency. J. Enzym. Inhib. Med. Chem. 23, 574-578 (2008).

36. Zhang, H., Forman, H. J. \& Choi, J. Gamma-glutamyl transpeptidase in glutathione biosynthesis. Methods Enzymol. 401, 468-483 (2005).

37. Smeyne, M. \& Smeyne, R. J. Glutathione metabolism and Parkinson's disease. Free Radic. Biol. Med. 62, 13-25 (2013).

38. Kumar, J. P. The fly eye: through the looking glass. Dev. Dyn. 247, 111-123 (2018).

39. Hauser, R. A. Levodopa: past, present, and future. Eur. Neurol. 62, 1-8 (2009).

40. Dorszewska, J., Prendecki, M., Lianeri, M. \& Kozubski, W. Molecular effects of Ldopa therapy in Parkinson's disease. Curr. Genomics 15, 11-17 (2014).

41. Slivka, A. \& Cohen, G. Hydroxyl radical attack on dopamine. J. Biol. Chem. 260, 15466-15472 (1985).

42. Fornstedt, B., Pileblad, E. \& Carlsson, A. In vivo autoxidation of dopamine in guinea pig striatum increases with age. J. Neurochem. 55, 655-659 (1990).

43. Asanuma, M., Miyazaki, I. \& Ogawa, N. Dopamine- or L-DOPA-induced neurotoxicity: the role of dopamine quinone formation and tyrosinase in a model of Parkinson's disease. Neurotox. Res. 5, 165-176 (2003).

44. Van Laar, V. S., Dukes, A. A., Cascio, M. \& Hastings, T. G. Proteomic analysis of rat brain mitochondria following exposure to dopamine quinone: implications for Parkinson disease. Neurobiol. Dis. 29, 477-489 (2008).

45. Monzani, E. et al. Dopamine, oxidative stress and protein-quinone modifications in parkinson's and other neurodegenerative diseases. Angew. Chem. Int Ed. Engl. 58, 6512-6527 (2019).

46. Holmay, M. J. et al. N-Acetylcysteine boosts brain and blood glutathione in Gaucher and Parkinson diseases. Clin. Neuropharmacol. 36, 103-106 (2013).

47. Mursaleen, L., Somavarapu, S. \& Zariwala, M. G. Deferoxamine and curcumin loaded nanocarriers protect against rotenone-induced neurotoxicity. J. Parkinsons Dis. 10, 99-111 (2020).
48. Man Anh, H., Linh, D. M., My Dung, V. \& Thi Phuong Thao, D. Evaluating doseand time-dependent effects of vitamin c treatment on a Parkinson's disease fly model. Parkinsons Dis. 2019, 9720546 (2019).

49. Matthews, D. G. et al. Centella Asiatica improves memory and promotes antioxidative signaling in 5XFAD mice. Antioxidants 8, 630 (2019).

50. Orlando, G. et al. Inhibitory effects induced by Vicia faba, Uncaria rhyncophylla, and Glycyrrhiza glabra water extracts on oxidative stress biomarkers and dopamine turnover in HypoE22 cells and isolated rat striatum challenged with 6-hydroxydopamine. Antioxidants 8, 602 (2019).

51. Rachitha, P. et al. Chemical composition, antioxidant potential, macromolecule damage and neuroprotective activity of Convolvulus pluricaulis. J. Tradit. Complement. Med. 8, 483-496 (2018).

52. Siddiqui, N. A. et al. Neuropharmacological profile of extracts of aerial parts of Convolvulus pluricaulis Choisy in mice model. Open Neurol. J. 8, 11-14 (2014).

53. Balkrishna, A., Thakur, P. \& Varshney, A. Phytochemical profile, pharmacological attributes and medicinal properties of convolvulus prostratus-a cognitive enhancer herb for the management of neurodegenerative etiologies. Front. Pharm. 11, 171 (2020)

54. Kizhakke, P. A., Olakkaran, S., Antony, A., Tilagul, K. S. \& Hunasanahally, P. G. Convolvulus pluricaulis (Shankhapushpi) ameliorates human microtubuleassociated protein tau (hMAPtau) induced neurotoxicity in Alzheimer's disease Drosophila model. J. Chem. Neuroanat. 95, 115-122 (2019).

55. Fahn, S. The spectrum of levodopa-induced dyskinesias. Ann. Neurol. 47, S2-9; discussion S9-11 (2000).

56. Olanow, C. W. et al. A randomized, double-blind, placebo-controlled, delayed start study to assess rasagiline as a disease modifying therapy in Parkinson's disease (the ADAGIO study): rationale, design, and baseline characteristics. Mov. Disord. 23, 2194-2201 (2008)

57. Spinazzi, M., Casarin, A., Pertegato, V., Salviati, L. \& Angelini, C. Assessment of mitochondrial respiratory chain enzymatic activities on tissues and cultured cells. Nat. Protoc. 7, 1235-1246 (2012).

58. Linford, N. J., Bilgir, C., Ro, J. \& Pletcher, S. D. Measurement of lifespan in Drosophila melanogaster. J. Vis. Exp. 71, 50068 (2013). 